\title{
Fermion masses and mixings in $\mathrm{SO}(10)$ models and the neutrino challenge to supersymmetric grand unified theories
}

\author{
Stefano Bertolini and Thomas Schwett \\ Scuola Internazionale Superiore di Studi Avanzati, via Beirut 4, \\ I-34014 Trieste and INFN, Sezione di Trieste, Italy \\ Michal Malinsky猝 \\ School of Physics and Astronomy, University of Southampton, SO16 1BJ Southampton, United Kingdom \\ and CERN Theory Division, CH-1211 Geneva 23, Switzerland.
}

(Dated: April 28, 2006)

\begin{abstract}
We present a detailed study of the quark and lepton mass spectra in a $S O(10)$ framework with one $10_{H}$ and one $\overline{126}_{H}$ Higgs representations in the Yukawa sector. We consider in full generality the interplay between type-I and type-II seesaw for neutrino masses. We first perform a $\chi^{2}$ fit of fermion masses independent on the detailed structure of the GUT Higgs potential and determine the regions of the parameter space that are preferred by the fermion mass sum rules. We then apply our study to the case of the minimal renormalizable SUSY $S O(10)$ GUT with one $10_{H}$, one $\overline{126}_{H}$, one $126_{H}$, and one $210_{H}$ Higgs representations. By requiring that proton decay bounds are fulfilled we identify a very limited area in the parameter space where all fermion data are consistently reproduced. We find that in all cases gauge coupling unification in the supersymmetric scenario turns out to be severely affected by the presence of lighter than GUT (albeit $B-L$ conserving) states. We then conclusively show that the minimal supersymmetric $S O(10)$ scenario here considered is not consistent with data. The fit of neutrino masses with type-I and type-II seesaws within a renormalizable $S O(10)$ framework strongly suggests a non-SUSY scenario for gauge unification.
\end{abstract}

PACS numbers: 12.10.-g, 12.60.Jv, 14.60.Pq, 12.15.Ff

\section{INTRODUCTION}

Understanding the pattern of fermion masses and mixings is one of the longstanding challenges in particle physics. In this respect Grand Unified Theories (GUTs) do provide an appealing and powerful tool to address the multiplicity of matter states, by involving stringent relations among the known fermions (and possibly implying a natural enlargement of the minimal sector). Very appealing candidates for a GUT are models based on the $S O(10)$ gauge group [1]. All the known fermions plus righthanded neutrinos fit into three 16-dimensional spinorial representations of $S O(10)$, and hence the model naturally leads to neutrino masses based on the seesaw mechanism [2, 3].

In this work we concentrate on a minimal renormalizable version of the supersymmetric $S O(10)$ GUT, where fermion mass matrices are obtained from the Yukawa couplings of the matter fields to 10 and 126 dimensional Higgs representations [4]. The $\overline{126}_{H}$ representation contains the scalar multiplets $(10,3,1) \oplus(\overline{10}, 1,3)$ under the Pati-Salam subgroup [5] $S U(4)_{C} \otimes S U(2)_{L} \otimes S U(2)_{R}$, whose vacuum expectation values (VEVs) generate mass matrices

*Electronic address: bertolin@sissa.it

$\dagger$ Electronic address: schwetz@sissa.it

‡Electronic address: malinsky@phys.soton.ac.uk for the left- and right-handed neutrino fields respectively. Hence, in general there are contributions to the effective mass matrix of the light neutrinos from the type-I [2] as well as type-II [3] seesaw mechanisms. Thanks to its minimality the model leads to constrained relations among quark and lepton mass matrices [6, 7]:

$$
\begin{aligned}
M_{d} & =v_{d}^{10} Y_{10}+v_{d}^{126} Y_{126}, \\
M_{u} & =v_{u}^{10} Y_{10}+v_{u}^{126} Y_{126}, \\
M_{\ell} & =v_{d}^{10} Y_{10}-3 v_{d}^{126} Y_{126}, \\
M_{D} & =v_{u}^{10} Y_{10}-3 v_{u}^{126} Y_{126}, \\
M_{L} & =v_{L} Y_{126}, \\
M_{R} & =v_{R} Y_{126} .
\end{aligned}
$$

Here $M_{D}, M_{L}, M_{R}$ denote the Dirac neutrino mass matrix, the mass matrix of the light left-handed neutrinos, and the mass matrix of the heavy righthanded neutrinos respectively. The indices $d, u$ refer to down- and up-quarks, $\ell$ denotes the charged leptons, while $Y_{10}$ and $Y_{126}$ are two complex symmetric matrices related to the $10_{H}$ and $\overline{126}_{H}$ Yukawa interactions. The various $v$ 's denote the VEVs of the relevant Higgs multiplets, with $v_{u, d}^{10,126}$ standing for the $S U(2)_{L}$ doublet components giving rise to the light MSSM-like Higgses, while $v_{R}\left(v_{L}\right)$ are the $S U(2)_{L}$ singlet (triplet) VEVs entering the type-I (type-II) seesaw formulae for the light neutrino masses. The effective mass matrix for the light neutrinos is then written as

$$
M_{\nu}=M_{L}-M_{D} M_{R}^{-1} M_{D},
$$

where the symmetry of $M_{D}$ is used. In Eq. (2) the 
first and second terms correspond to type-II and type-I seesaws respectively. Hence, all fermion mass matrices are predicted in terms of two complex symmetric Yukawa matrices and six VEVs.

Quite an amount of activity has been devoted recently to the study of the minimal renormalizable SUSY $S O(10)$ model, see for instance Refs. 8, 9, $10,11,12,13,14,15,16,17,18,19,20,21,22$, $23,24,25,26]$. This interest has been triggered to some extent by the experimental progress in neutrino physics, and the observation of Refs. [10, 11], that within the minimal $S O(10)$ model with typeII seesaw dominance $b-\tau$ unification allows naturally for a large lepton mixing due to the destructive interference between the 33 elements of the downquark and charged lepton mass matrices. Analyses of proton decay within this setting are found, e.g., in Refs. [27, 28], while various extensions of the model have been considered in view of rising some of the tensions with the data, by adding nonrenormalizable terms [17], by minimally extending the Higgs sector [18], or by including Yukawa couplings of the fermion fields with an additional 120dimensional Higgs representation [23, 24, 26].

Most of the previously quoted analyses consider the dominance of one type of seesaw and assume that the vacuum gives the correct neutrino mass scale. Very recently two studies appeared showing an intrinsic antagonism between seesaw neutrino masses and coupling unification in the minimal SUSY $S O(10)$ scenario [29, 30], pointing out a critical tension between the needed seesaw neutrino scale and the detailed spectrum arising from the minimal $S O(10)$ breaking GUT vacuum [15, 19, 31].

The aim of the present paper is twofold. First, in Secs. II and III we study in detail the implications of the fermion mass sum rules that emerge from $10_{H}$ and $\overline{126}_{H}$ Yukawa terms in a $S O(10)$ GUT framework. The analysis is performed in full generality, allowing for complex Yukawas and VEVs, and considering the neutrino mass matrix as originating from an admixture of type-I and type-II seesaw as given by Eq. (2). In the second part of the paper, Sec. IV] we specialize our study to the minimal renormalizable SUSY $S O(10)$ model, by including the constraints coming from the detailed structure of the GUT symmetry breaking vacuum and performing an exhaustive study of the parameter space by optimizing a $\chi^{2}$ function. This approach is complementary to the random parameter searches applied in previous studies, since the algorithm converges to an optimal solution (if it exists), and isolated solutions (allowed by an acceptable fine tuning of the parameters) can be found, which are easily missed in a random parameter scan.

We show conclusively that the minimal renormalizable $S O(10)$ supersymmetric scenario does not allow for a consistent fit of the fermion mass spectrum, the model being unable to reproduce the correct neutrino mass scale via type-I and/or type-II seesaw. Our negative result can be cast in more general (and simple) terms considering that, while i) the present constraints on proton decay force any GUT scale to lie above $10^{16} \mathrm{GeV}$ and ii) SUSY gauge unification works very well considering a desert scenario between the weak and the GUT scales, the neutrino mass coming from type-I and/or type-II seesaws, being proportional via Yukawa and Higgs potential couplings to $m_{\text {weak }}^{2} / M$, requires for $m_{\text {weak }} \approx$ $200 \mathrm{GeV}, M<10^{15} \mathrm{GeV}$. As a consequence (barring a strong interacting sector in the theory), intermediate mass scales appear which are bound to affect SUSY gauge coupling unification at the least.

All this applies to the minimal renormalizable setup. As it was pointed out very recently [32], nonminimal realizations of the renormalizable Yukawa sector such as those containing an additional 120dimensional Higgs multiplet may provide a way out of the issues: the neutrino mass scale can be enhanced by the type-I seesaw with right-handed neutrino masses below the GUT scale due to tiny Yukawa couplings associated to the $\overline{126}_{H}$ multiplet, whose role in the charged matter sector can be taken over by the new $120_{H}$ multiplet. Although the simplest realization of this programme (where the charged matter sector is dominated by $10_{H}$ and $120_{H}$ and the Yukawas of $\overline{126}_{H}$ are neglected) seems to fail [33], this attempt does provide a direction for further studies. Alternatively, Planck induced nonrenormalizable operators may also provide the scale suppression needed by the neutrino sector, see for instance Ref. [34] and references therein.

On the other hand, the simplicity and the high level of predictivity that makes the minimal renormalizable SUSY $S O(10)$ GUT extremely appealing is lost in both extensions. Before calling the minimal setup a dead end an extensive analysis of potential loopholes in the abovementioned arguments is due. This aim we pursue with the present paper up to, helas, the bitter end.

\section{DESCRIPTION OF THE ANALYSIS}

\section{A. The parameterization}

In order to investigate whether Eqs. (11) and (2) allow for fermion masses and mixing in agreement with the data we proceed as follows. It turns out to be convenient to express the $Y_{10}$ and $Y_{126}$ Yukawa matrices in terms of $M_{\ell}$ and $M_{d}$, and substitute them in the expressions for $M_{u}, M_{D}$ and $M_{\nu}$ :

$$
\begin{aligned}
M_{u} & =f_{u}\left[(3+r) M_{d}+(1-r) M_{\ell}\right], \\
M_{D} & =f_{u}\left[3(1-r) M_{d}+(1+3 r) M_{\ell}\right],
\end{aligned}
$$


where

$$
f_{u}=\frac{1}{4} \frac{v_{u}^{10}}{v_{d}^{10}}, \quad r=\frac{v_{d}^{10}}{v_{u}^{10}} \frac{v_{u}^{126}}{v_{d}^{126}} .
$$

The neutrino mass matrix is obtained as

$$
M_{\nu}=f_{\nu}\left[\left(M_{d}-M_{\ell}\right)+\xi \frac{M_{D}}{f_{u}}\left(M_{d}-M_{\ell}\right)^{-1} \frac{M_{D}}{f_{u}}\right],
$$

with

$$
f_{\nu}=\frac{1}{4} \frac{v_{L}}{v_{d}^{126}}, \quad \xi=-\frac{\left(4 f_{u} v_{d}^{126}\right)^{2}}{v_{L} v_{R}} .
$$

The parameter $|\xi|$ controls the relative importance of the type-I and type-II seesaw terms: For $|\xi| \rightarrow 0$ one obtains pure type-II seesaw, whereas $|\xi| \rightarrow \infty$ (with $f_{\nu}|\xi| \simeq$ const.) corresponds to type-I seesaw. For $|\xi| \sim 1$ both contributions are comparable.

In what follows we denote diagonal mass matrices by $\hat{m}_{x}, x=u, d, \ell, \nu$, with eigenvalues corresponding to the particle masses, i.e., being real and positive. We choose a basis where the down-quark matrix is diagonal: $M_{d}=\hat{m}_{d}$. In this basis $M_{\ell}$ is a general complex symmetric matrix, that can be written as $M_{\ell}=W_{\ell}^{\dagger} \hat{m}_{\ell} W_{\ell}^{*}$, where $W_{\ell}$ is a general unitary matrix. Without loss of generality $f_{u}$ and $f_{\nu}$ can be taken to be real and positive. Hence, the independent parameters are given by 3 down-quark masses, 3 charged lepton masses, 3 angles and 6 phases in $W_{\ell}, f_{u}, f_{\nu}$, together with two complex parameters $r$ and $\xi: 21$ real parameters in total, among which 8 phases. Using Eqs. (3), (4), and (6) all observables (6 quark masses, 3 CKM angles, 1 CKM phase, 3 charged lepton masses, 2 neutrino mass-squared differences, the mass of the lightest neutrino, and 3 PMNS angles, 19 quantities altogether) can be calculated in terms of these input parameters. Although the number of parameters is larger than the number of observabels the system is sensibly overconstrained due to the non-linear structure of the problem.

Since we work in a basis where the down-quark mass matrix is diagonal the CKM matrix is given by the unitary matrix diagonalizing the up-quark mass matrix up to diagonal phase matrices:

$$
\hat{m}_{u}=W_{u} M_{u} W_{u}^{T}
$$

with

$$
W_{u}=\operatorname{diag}\left(e^{i \beta_{1}}, e^{i \beta_{2}}, e^{i \beta_{3}}\right) V_{\mathrm{CKM}} \operatorname{diag}\left(e^{i \alpha_{1}}, e^{i \alpha_{2}}, 1\right),
$$

where $\alpha_{i}, \beta_{i}$ are unobservable phases at low energy. The neutrino mass matrix given in Eq. (6) is diagonalized by $\hat{m}_{\nu}=W_{\nu} M_{\nu} W_{\nu}^{T}$, and the PMNS matrix is determined by $W_{\ell}^{*} W_{\nu}^{T}=\hat{D}_{1} V_{\mathrm{PMNS}} \hat{D}_{2}$, where $\hat{D}_{1}$ and $\hat{D}_{2}$ are diagonal phase matrices similar to those in Eq. (9).

\begin{tabular}{lr}
\hline \hline observable & input data \\
\hline$m_{d}[\mathrm{MeV}]$ & $1.24 \pm 0.41$ \\
$m_{s}[\mathrm{MeV}]$ & $21.7 \pm 5.2$ \\
$m_{b}[\mathrm{GeV}]$ & $1.06_{-0.09}^{+0.14}$ \\
$m_{u}[\mathrm{MeV}]$ & $0.55 \pm 0.25$ \\
$m_{c}[\mathrm{MeV}]$ & $210 \pm 0.21$ \\
$m_{t}[\mathrm{GeV}]$ & $82.4_{-14.8}^{+30.3}$ \\
$\sin \phi_{23}^{\mathrm{CKM}}$ & $0.0351 \pm 0.0013$ \\
$\sin \phi_{13}^{\mathrm{CKM}}$ & $0.0032 \pm 0.0005$ \\
$\sin \phi_{12}^{\mathrm{CKM}}$ & $0.2243 \pm 0.0016$ \\
$\delta_{\mathrm{CKM}}$ & $60^{\circ} \pm 14^{\circ}$ \\
$\sin { }^{2} \theta_{23}^{\mathrm{PMNS}}$ & $0.50 \pm 0.065$ \\
$\sin ^{2} \theta_{13}^{\mathrm{PMNS}}$ & $<0.0155$ \\
$\sin ^{2} \theta_{12}^{\mathrm{PMNS}}$ & $0.31 \pm 0.025$ \\
$\Delta m_{21}^{2}\left[\mathrm{eV}^{2}\right]$ & $(7.9 \pm 0.3) 10^{-5}$ \\
$\left|\Delta m_{31}^{2}\right|\left[\mathrm{eV}^{2}\right]$ & $\left(2.2_{-0.27}^{+0.37}\right) 10^{-3}$ \\
\hline \hline
\end{tabular}

TABLE I: Sample of GUT scale input data used in this work (central values and $1 \sigma$ errors) for $M_{\mathrm{SUSY}}=1 \mathrm{TeV}, \tan \beta=$ 10 , and $M_{\mathrm{GUT}}=2 \times 10^{16} \mathrm{GeV}$ (see text for details and references). Charged lepton masses are listed in Eq. (10).

\section{B. Input data and $\chi^{2}$ analysis}

As input data we use quark and lepton masses and mixing angles evaluated at the GUT scale, based on the RGE analysis of Ref. [35]. As a typical example we consider a SUSY scale $M_{\mathrm{SUSY}}=1 \mathrm{TeV}, \tan \beta=$ 10 , and a GUT scale $M_{\mathrm{GUT}}=2 \times 10^{16} \mathrm{GeV}$. Since charged lepton masses are known with an accuracy of better than $10^{-3}$ we do not consider them as free parameters but fix them to the (GUT scale) central values from Ref. [35]:

$$
\begin{aligned}
& m_{e}=0.3585 \mathrm{MeV}, \\
& m_{\mu}=75.67 \mathrm{MeV}, \\
& m_{\tau}=1292.2 \mathrm{MeV} .
\end{aligned}
$$

The remaining data are listed in Tab. I. For the heavy quark masses $m_{c}, m_{b}, m_{t}$ we adopt the values and uncertainties used in Ref. [35], whereas for the light quarks we update the data to the ranges given in Ref. [36]: $m_{u}=1.5-4 \mathrm{MeV}, m_{d}=4-8 \mathrm{MeV}$, $m_{s}=80-130 \mathrm{MeV}(\overline{\mathrm{MS}}$ masses at $2 \mathrm{GeV})$. The corresponding values at the GUT scale are given in Tab. [1. together with the CKM angles and phase [24].

Concerning the neutrino parameters, we note that in the setup under consideration the neutrino mass spectrum is generally normal and hierarchical with $m_{1}<m_{2}<m_{3}$. In this case the RGE running of the PMNS angles [37, 38] is of order $10^{-5}\left(1+\tan ^{2} \beta\right) \sim$ $10^{-3}$, and therefore negligible to a good approximation. The running of the neutrino masses is small as well (a running effect common to all three neutrino masses can be absorbed in the free parameter $f_{\nu}$, 
compare Eq. (66)). In our analysis we use the low energy neutrino parameters, as obtained from recent global fits to neutrino oscillation data [39, 40]. We do not include any constraint on the lightest neutrino mass $m_{1}$, since the values that are obtained are much below the sensitivity of the present data.

Let us denote the central values and errors of the observables by $O_{i}$ and $\sigma_{i}$, where $i=1, \ldots, 15$ runs over all the quantities listed in Tab. I. As described above, the predictions of these observables, $P_{i}$, depend on the parameters $x_{\alpha}$, where $\alpha=1, \ldots, 18$ runs over 3 down-quark masses, 9 real parameters in $W_{\ell}$, $f_{u}, f_{\nu},|r|, \arg (r),|\xi|, \arg (\xi) .{ }^{1}$ Then a $\chi^{2}$-function is constructed as

$$
\chi^{2}\left(x_{\alpha}\right)=\sum_{i=1}^{15}\left(\frac{P_{i}\left(x_{\alpha}\right)-O_{i}}{\sigma_{i}}\right)^{2} .
$$

The data are fitted by minimizing this function with respect to the parameters $x_{\alpha}$. The minimization of the scale factors $f_{u}$ and $f_{\nu}$ can be done analytically. The remaining 16 dimensional minimization is performed with an algorithm based on the so-called down-hill simplex method [41].

Let us note that the minimization is technically rather challenging. The problem involves parameters which differ by many orders of magnitude, and some of the solutions are extremely fine-tuned, which leads to very steep valleys in the $\chi^{2}$ landscape. Moreover, due to the high dimensionality and the non-linearity of the problem there is a large number of local minima. In the numerical analysis much effort has been devoted to find the absolute minimum, involving random methods and dedicated investigations to each particular problem under consideration. However, as it is well known, by numerical methods it is difficult to assess with absolute confidence that an absolute minimum has been found. Although each minimum has been carefully tested for possible improvements one should always keep in mind the possibility that a better solution might exist somewhere in the parameter space.

\section{GENERAL FIT OF FERMION MASSES AND MIXINGS}

For the discussion of the obtained fits we classify the solutions by the values of the parameter $|\xi|$ which controls the relative weight of type-I and type-II seesaw mechanism. In Fig. 1 the $\chi^{2}$ minimum is shown as a function of this parameter. This means that for

\footnotetext{
1 Since we fix the charged lepton masses to the values given in Eq. (10) they are neither included in the set of observables $O_{i}$, nor among the parameters $x_{\alpha}$.
}

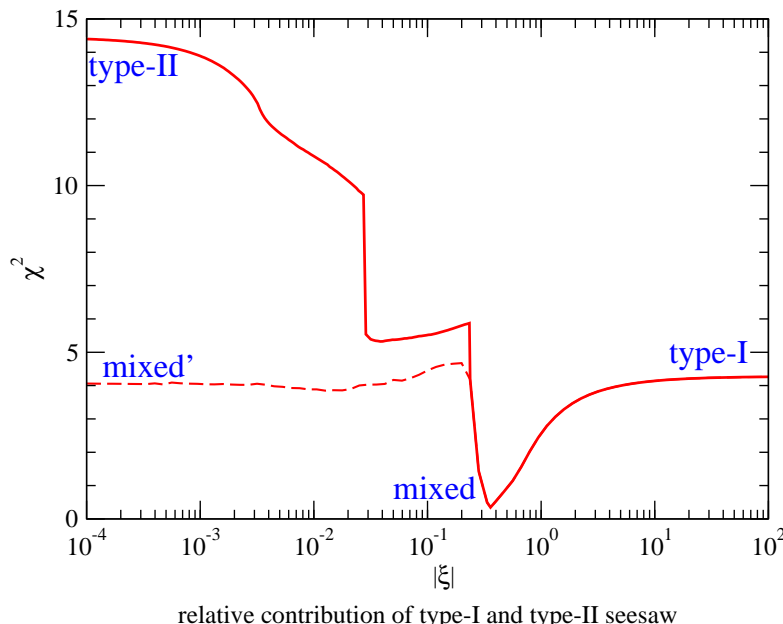

FIG. 1: $\chi^{2}$ as a function of the parameter $|\xi|$ which controls the relative relevance of type-I and type-II seesaw terms. The dashed curve corresponds to the singular (fine tuned) solution denoted by mixed' (see text).

fixed $|\xi|, \chi^{2}\left(x_{\alpha}\right)$ is minimized with respect to all the other parameters $x_{\alpha}$ with $\alpha \neq|\xi|$. In Tab. II some parameter values and the predictions for the data are given for four sample points.

One of the main results of this work is clearly visible in Fig. 1] We find a pronounced minimum for $|\xi| \simeq 0.36$ which corresponds to a mixture of type-I and type-II seesaw with a comparable size of both terms. Such a "mixed" solution provides an excellent fit to the data with $\chi^{2} \approx 0.35$. From the results given in Tab. II in the column labeled "mixed" it is seen that all observables are fitted within $\lesssim 0.4 \sigma$. In particular, all the lepton mixing angles and the neutrino mass-squared differences are very close to their experimental values. Also CKM CP-violation is described correctly by the value $\delta_{\mathrm{CKM}}=61^{\circ}$ obtained in this solution. We conclude that a scenario with both seesaw terms of comparable size allows for an excellent description of fermion masses and mixings, confirming the results of Ref. [25]. Whether this solution is viable still depends on the detailed study of the global vacuum of the given $S O(10)$ model.

The $\chi^{2}$ increases by increasing $|\xi|$, and it approaches a value of $\chi^{2} \simeq 4.3$ for $|\xi| \gtrsim 10$, when the neutrino mass matrix becomes dominated by the type-I seesaw term. Also in the case of complete type-I dominance the fit is very good, with most observables within $\lesssim 0.3 \sigma$, with the sole exception of the down-quark mass $m_{d}$ which shows a $-1.87 \sigma$ deviation from its prediction (see Tab. II, column "type-I"). As we will discuss in more detail later, a low value of $m_{d}$ is required for a valuable type-I seesaw fit. Let us note that all neutrino parameters are in excellent agreement with the observations, and in particular, the correlation between $\theta_{12}^{\mathrm{PMNS}}, \theta_{23}^{\mathrm{PMNS}}$, and the ratio $\Delta m_{31}^{2} / \Delta m_{21}^{2}$ found in Ref. [25] seems 


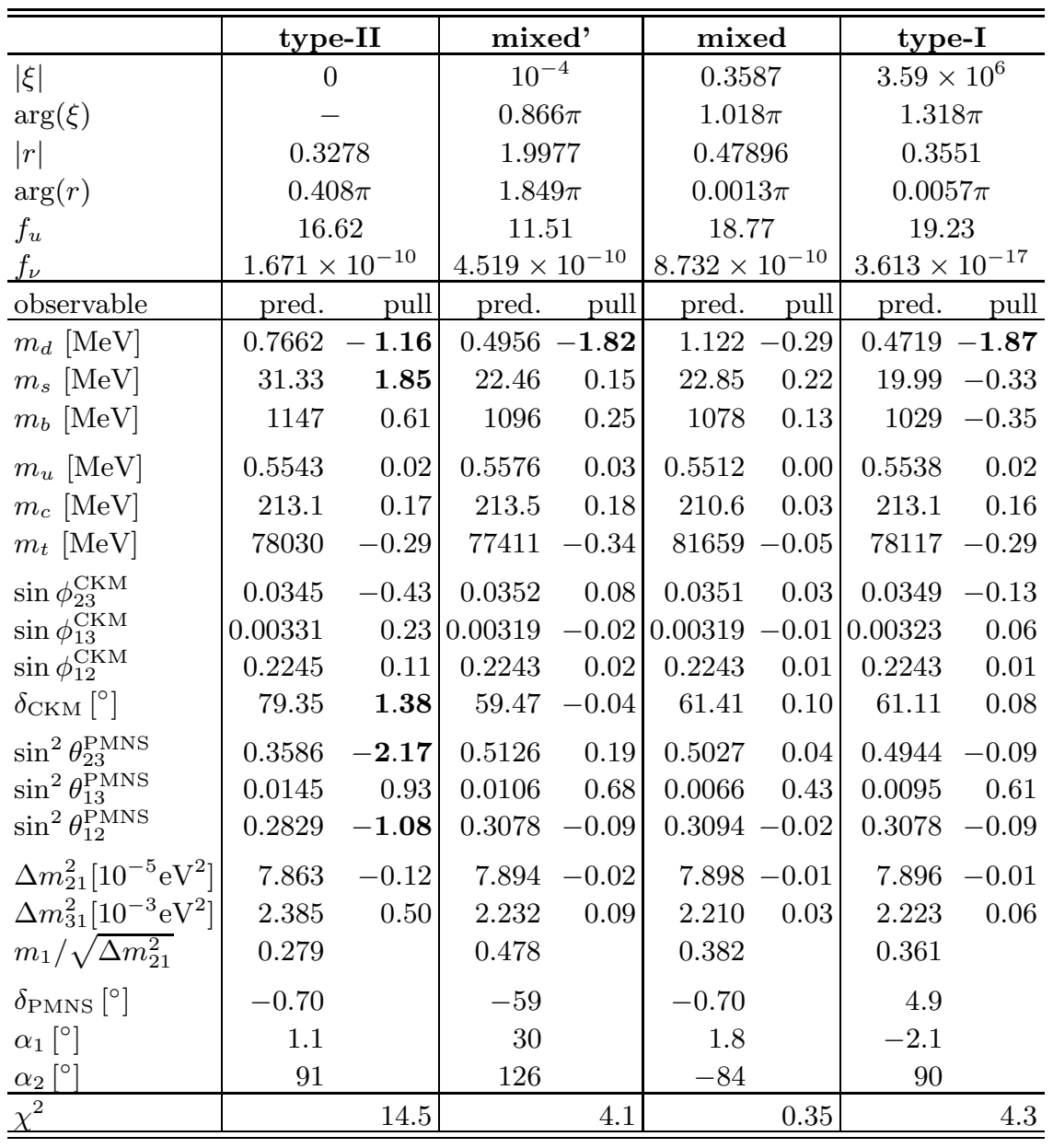

TABLE II: Parameter values and predictions in four example solutions corresponding to different terms dominating the neutrino mass matrix: type-I, type-II, or both contributions of comparable size (mixed and mixed'). In the column "pred." the predicted values $P_{i}$ for the observables are given, the column "pull" shows the number of standard deviations from the observations, $\left(P_{i}-O_{i}\right) / \sigma_{i}$, using the data and errors from Tab. [ Deviations of more than $1 \sigma$ are highlighted in boldface. The final $\chi^{2}$ is the sum of the squares of the numbers in the "pull" column. See the text for comments on the values of the leptonic CP phases.

not to apply here. This solution is not plagued by the problem of accomodating the correct $\delta_{\mathrm{CKM}}$ phase found in Ref. [17], and discussed on general grounds in Ref. 24].

For a pure type-II solution with $|\xi|=0$ there is more tension in the fit. Although a $\chi^{2} \simeq 14.5$ might be acceptable for 15 data points from a statistical point of view, several observables are 1 to $2 \sigma$ away from the central value. Among all, one needs a large value of the strange-quark mass, namely $m_{s} \simeq 31 \mathrm{MeV}$ that is $1.85 \sigma$ too large, while the PMNS angle $\theta_{23}$ is too small by $2.2 \sigma$. Furthermore, $m_{d}$, the CKM phase, and the PMNS angle $\theta_{12}$ show a pull greater than $1 \sigma$. These results agree with previous analyses of pure type-II solutions [14, 24, 25].

The dashed line in Fig. 1 1 corresponds to an interesting variant of a solution with comparable typeI and type-II contributions. Formally this solution has a rather small value of $|\xi|$, which would signal type-II dominance. However, in this case one eigen- value of $\left(M_{d}-M_{\ell}\right)$ is very small, i.e., this matrix is close to singular, which implies that its inverse has large entries. As a consequence the second term in Eq. (6) turns out to be comparable to the first term, in spite of the small value of $|\xi|$. Let us denote the type-II term in Eq. (6) by $M^{\mathrm{II}}$ and the type-I term by $M^{\mathrm{I}}$. Then for the solution denoted mixed' in Fig. 1 and Tab. II we find that the matrix entries are of a similar size: $0.2 \lesssim\left|M_{i j}^{\mathrm{I}} / M_{i j}^{\mathrm{II}}\right| \lesssim 1.5$ for all $i, j=1,2,3$. Obviously this solution involves a very precise tuning between the $M_{d}$ and $M_{\ell}$ matrices such that the difference becomes close to singular. Changing the input values for the down-quark masses and the charged lepton mixing matrix $W_{\ell}$ by a factor of $\left(1+10^{-4}\right)$ destroys in general the fit and leads to $\chi^{2}$ values of order $100 .^{2}$ Let us add that we

2 The type-I, type-II, and mixed solutions require tuning of 


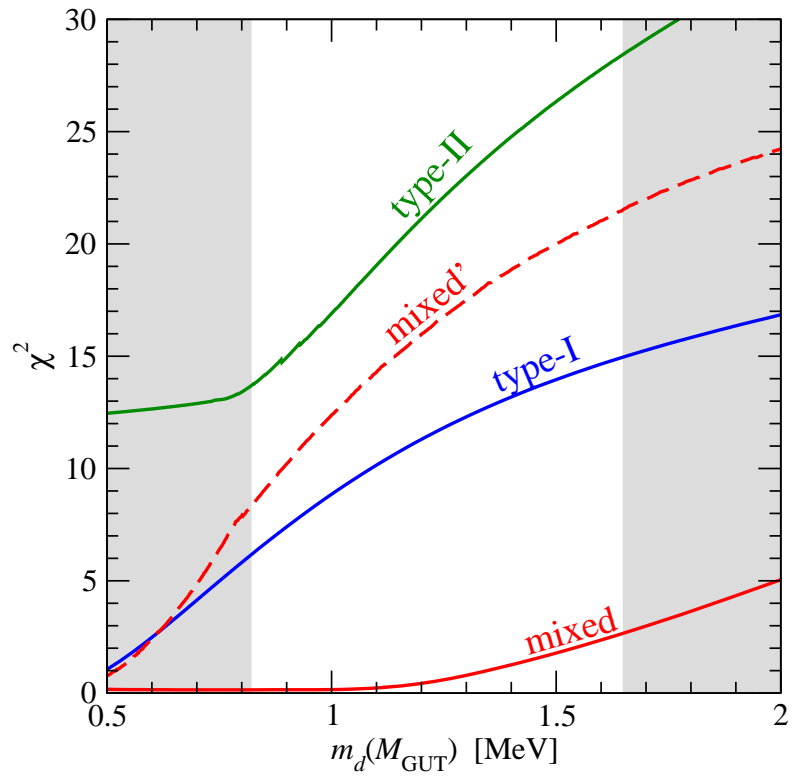

FIG. 2: $\chi^{2}$ as a function of $m_{d}$ for the type-I, type-II, mixed, and mixed' solutions given in Tab. III The unshaded region corresponds to the $1 \sigma$ interval for $m_{d}$ from Tab. \

obtain mixed' type solutions by extrapolating from $|\xi|=10^{-4}$ down to very small values of $|\xi| \lesssim 10^{-9}$. However, at some point these results are likely to become unreliable, since the numerical inversion of a nearly singular matrix is limited by the accuracy of the algorithm.

\section{A. The role of $m_{d}$}

The down-quark mass plays a relevant role in obtaining a good fit to the data. As it is visible from Tab. III the type-II, mixed', and type-I solutions require values of $m_{d}$ which are about $1.2 \sigma, 1.8 \sigma$, and $1.9 \sigma$ below the preferred value given in Tab. II. In Fig. 2 we illustrate how the fits become worse when $m_{d}$ is increased. Especially the very good fits of the type-I and mixed' solutions with $\chi^{2} \simeq 4$ are strongly affected by increasing $m_{d}$, and values of $m_{d} \simeq 1.5 \mathrm{MeV}$ at $M_{\mathrm{GUT}}$ lead to $\chi^{2} \simeq 15$ and 20 , respectively. Also for the pure type-II case the fit soon becomes unacceptable if $m_{d}$ is increased. Only the mixed solution has enough freedom to accomodate larger values of the down-quark mass, and in this case also a very good fit is possible for $m_{d} \simeq 2 \mathrm{MeV}$.

As a matter of fact, it is quite easy to understand these results on an analytical basis. As it was pointed out in Refs. [14] and 24] the quality of the

the parameters with a typical accuracy of better than $10^{-3}$ (the tuning is slightly less severe for the type-II case). charged sector fit is gauged by the need to reproduce the tiny electron mass that obeys an approximate formula of the form [24]:

$$
\begin{aligned}
\left|k^{\prime} \tilde{m}_{e}\right| \mathrm{e}^{i \psi}= & -|\tilde{r}| \mathrm{e}^{i \beta_{1}} F_{d} \lambda^{4}+\mathrm{e}^{i \alpha_{2}} F_{c} \lambda^{6} \\
& -A^{2} \Lambda^{2} \mathrm{e}^{i \alpha_{3}} \lambda^{6} \frac{|\tilde{r}|}{\mathrm{e}^{i \alpha_{3}}-|\tilde{r}|}+\mathcal{O}\left(\lambda^{7}\right)
\end{aligned}
$$

where $\tilde{m}_{e} \equiv m_{e} / m_{\tau}, \Lambda \equiv 1-\rho-i \eta(\rho, \eta$ being the Wolfenstein CKM parameters and $\lambda$ the Cabibbo angle), while $F_{d} \equiv \frac{m_{d}}{m_{b}} / \lambda^{4}, F_{c} \equiv \frac{m_{c}}{m_{t}} / \lambda^{4}$ are $\mathcal{O}(1)$ factors. The CKM phases $\alpha_{i}, \beta_{i}$ are defined in Ref. [24]. The parameters $\tilde{r}$ and $k^{\prime}$ are given by

$$
\left|k^{\prime}\right|=\frac{m_{\tau}}{m_{t}} f_{u}|r-1|, \quad|\tilde{r}|=\frac{m_{b}}{m_{t}} f_{u}|r+3| .
$$

It is clear that in order to get near the physical value $\tilde{m}_{e} \sim 2.5 \times 10^{-4}$ for $\left|k^{\prime}\right| \sim 0.25$ (as suggested by the relevant trace identities), the dominant first term on the RHS of Eq. (12) must be either strongly suppressed (leading to the observed effect of low $m_{d}$ preference) or cancelled to a large extent by the subleading ones (often at odds with the CKM phase in the first quadrant, c.f. [14, 24]). Therefore, for low values of $m_{d}$ the available portion of the parameter space is larger and allows for a better global fit of the remaining physical parameters. It is perhaps worth mentioning that there is another pattern in our data (though by far much weaker than the low $m_{d}$ preference) that can be justified on the same grounds, namely a generic drift towards lower $m_{t}$ and higher $m_{c}$ regions. One verifies that in such a case the subdominant term proportional to $F_{c}$ gets larger and allows for a better "screening" of the dominant first term on the RHS of formula (12).

\section{B. Predictions for the neutrino parameters}

In this section we discuss in some detail the predictions of the studied setup for the neutrino sector. Our main results are summarized in Fig. 3, where we show how the $\chi^{2}$ changes if $\theta_{23}^{\text {PMNS }}, \theta_{13}^{\text {PMNS }}$, and the mass of the lightest neutrino $m_{1}$ are varied. Technically this analysis is performed in the following way: to test the variation of an observable $O_{k}$ the term with $i=k$ is removed from the $\chi^{2}$ given in Eq. (11) (this ensures that the only constraint on the observable comes from the mass sum rules and not from the input data). Then, in order to test a certain value $O^{*}$ for the observable $O_{k}$ a term is added to the $\chi^{2}$ with a very small error of $1 \%$, to confine the fit: $\left(P_{k}\left(x_{\alpha}\right)-O^{*}\right)^{2} /\left(0.01 O^{*}\right)^{2}$. After the minimization this term is removed, and the $\chi^{2}$ is evaluated at the point obtained in the minimization.

Fig. 3 (left) shows the constraint on the PMNS mixing angle $\theta_{23}^{\mathrm{PMNS}}$. One can see that for the typeI, mixed, and mixed' solutions there is no definite 

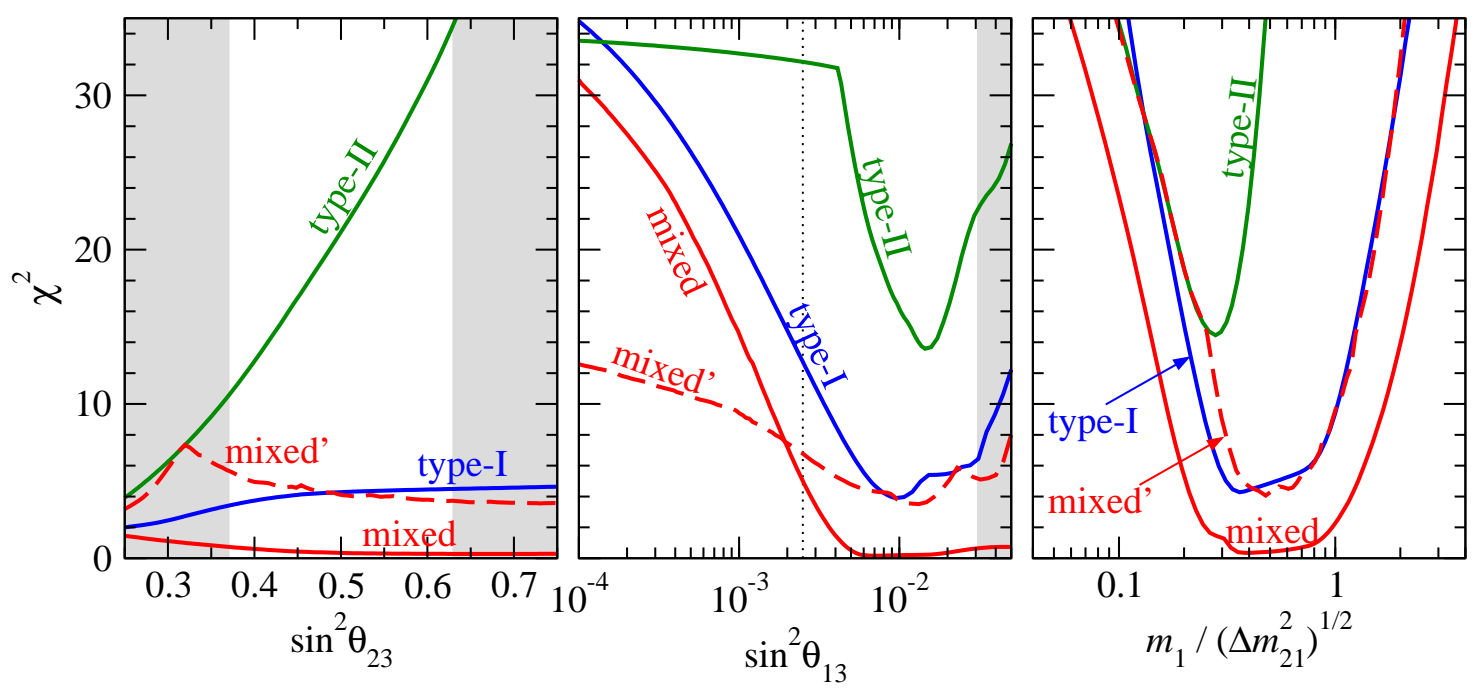

FIG. 3: $\chi^{2}$ as a function of $\sin ^{2} \theta_{23}^{\mathrm{PMNS}}$ (left), $\sin ^{2} \theta_{13}^{\mathrm{PMNS}}$ (middle) and of $R \equiv m_{1} / \sqrt{\Delta m_{21}^{2}}$ (right) for the type-I, type-II, mixed, and mixed' solutions given in Tab. [I] The shaded regions are excluded at $2 \sigma$ according to the data given in Tab. [I] The dotted vertical line shows roughly the sensitivity to $\theta_{13}^{\text {PMNS }}$ of neutrino oscillation experiments within a timescale of 10 years [43].

prediction for this angle and very good fits are possible with values of $\theta_{23}^{\mathrm{PMNS}}$ in the whole range allowed by the data. In contrast a scenario with pure type-II seesaw shows a clear preference for small values of $\sin ^{2} \theta_{23}^{\mathrm{PMNS}}$. In particular, maximal mixing $\sin ^{2} \theta_{23}^{\mathrm{PMNS}}=0.5$ is disfavoured with respect to the best fit value $\sin ^{2} \theta_{23}^{\mathrm{PMNS}}=0.36$ with $\Delta \chi^{2} \approx 11$. Hence, the pure type-II seesaw model predicts sizable deviations from maximal mixing within the reach of upcoming neutrino oscillation experiments, see, e.g., Refs. 40, 42]. This result is in agreement with Refs. [24, 25]. Let us stress, however, that deviations from maximal mixing are not a general prediction of the $S O(10)$ model under consideration; it holds only for the pure type-II case.

Concerning the mixing angle $\theta_{13}^{\mathrm{PMNS}}$, for all solutions the best fit point predicts values close to the present upper bound and clearly within the reach of upcoming neutrino oscillation experiments [40, 43]. Also in this case the pure type-II solution gives the most stringent prediction. However, if the fit is stretched to some degree, the type-I and mixed solutions allow also for smaller values of $\theta_{13}^{\mathrm{PMNS}}$ that might be difficult to detect in the next round of experiments. For instance, in the mixed solution case a fit with $\chi^{2} \approx 7$ is possible for $\sin ^{2} \theta_{13}^{\mathrm{PMNS}}=2 \times 10^{-3}$. An interesting feature of the mixed' solution is that even for very tiny values of $\sin ^{2} \theta_{13}^{\mathrm{PMNS}} \lesssim 10^{-4} \mathrm{a}$ reasonable fit can be obtained with $\chi^{2} \approx \tilde{12}$.6. The main contributions to the $\chi^{2}$ in this case are deviations of $-1.7 \sigma$ for $m_{d},-1.2 \sigma$ for $m_{s},-2.0 \sigma$ for $m_{t}$, and $+1.4 \sigma$ for $\sin ^{2} \theta_{23}^{\mathrm{PMNS}}$. The existence of this solution shows that if no signal of $\theta_{13}^{\mathrm{PMNS}}$ is detected by the upcoming experiments it might be still possible to construct models with viable predictions for fermion masses and mixings, although the amount of fine tuning will increase.

Another interesting information the setup under consideration provides, concerns the shape of the neutrino mass spectrum. The most solid prediction is the normal mass ordering, which means that $m_{1}<m_{2}<m_{3}$, i.e., $\Delta m_{31}^{2}>0$; no viable solution has been found for inverted ordering $\left(\Delta m_{31}^{2}<0\right)$ which is equally allowed by present oscillation data. The predictions for the absolute neutrino mass scale are given in terms of the ratio of the lightest neutrino mass $m_{1}$ to the square root of the "solar" masssquared difference:

$$
R \equiv \frac{m_{1}}{\sqrt{\Delta m_{21}^{2}}} .
$$

The best fit values for this ratio given in Tab. II] for the four example solutions are in the range $0.28 \leq$ $R \leq 0.48$. These values show that there is only a modest hierarchy in the neutrino masses. For example, a value of $R=0.3$ implies that $m_{2} \simeq 3.5 m_{1}$, i.e., $m_{1}$ and $m_{2}$ are of the same order of magnitude. From the plot in the right panel of Fig. 3 one can infer the allowed ranges for the ratio $R$. We find reasonable fits for values in the range

$$
0.2 \lesssim R \lesssim 2
$$

where the upper bound implies $m_{2} \simeq 1.1 m_{1}$. For the pure type-II solution the ratio $R$ is stronger constrained to values around the best fit point of 0.28 , whereas the constraint is weakest for the mixed solution. Note that a quasi-degenerate neutrino spectrum with $m_{1} \simeq m_{2} \simeq m_{3}$ would correspond to $R \gtrsim \sqrt{\Delta m_{31}^{2} / \Delta m_{21}^{2}} \simeq 5.3$ which is clearly excluded within the $S O(10)$ framework under consideration. 
The range for $R$ given in Eq. (15) implies the following intervals for $m_{1}$ and the sum of the neutrino masses $\Sigma \equiv m_{1}+m_{2}+m_{3}$ :

$$
\begin{gathered}
1.8 \times 10^{-3} \mathrm{eV} \lesssim m_{1} \lesssim 1.8 \times 10^{-2} \mathrm{eV} \\
0.058 \mathrm{eV} \lesssim \Sigma \lesssim 0.088 \mathrm{eV} .
\end{gathered}
$$

For comparison we note that for $m_{1}=0$ one has $\Sigma=0.056 \mathrm{eV}$, which shows that the smallest values of $m_{1}$, for which reasonable fits are found, are close to the case where the contribution of $m_{1}$ to $\Sigma$ can be neglected. ${ }^{3}$

For any given set of input parameters the values of the phases in the PMNS matrix are determined as well. We have investigated in the four cases of Tab. II the predictions for the Dirac CP phase $\delta_{\mathrm{PMNS}}$, as well as the two Majorana phases $\alpha_{1}$ and $\alpha_{2}$, defined in analogy with Eq. (9) in the leptonic sector. In all cases we find a correlation among the phases, such that

$$
\delta_{\mathrm{PMNS}} \approx-2 \alpha_{1} \approx-2 \alpha_{2}+\pi
$$

For the mixed, mixed', and type-I solutions there is no definite prediction for the values of the phases, and, given the correlations above, fits of comparable quality are found for all values of the phases in the $[0,2 \pi]$ range. Only in the case of pure type-II seesaw the fit prefers values of $\delta_{\mathrm{PMNS}} \approx 0, \alpha_{1} \approx 0(\pi)$, $\alpha_{2} \approx \pm \pi / 2$, in agreement with the results of Ref. [24] (the Majorana phases $\phi_{1,2}$ there reported are defined according to Ref. [37] as $-2 \alpha_{1,2}$ ). Moving $\delta_{\mathrm{PMNS}}$ from the preferred value to $\pm 60^{\circ}$ increases the $\chi^{2}$ by 10 units.

\section{THE MINIMAL SUSY $S O(10)$ GUT}

Having analyzed the implications on the fermion mass fit of the $10_{H}$ plus $\overline{126}_{H} S O(10)$ Yukawa sector we focus now on the study of the so called minimal renormalizable SUSY SO(10) GUT [13]. The model is characterized (in addition to $10_{H}$ and $\overline{126}_{H}$ ) by the presence of the $126_{H}$ and $210_{H}$ representations in the Higgs sector. The $126_{H}$ is needed to preserve the GUT-scale D-flatness while $210_{H}$ plays the dual role of triggering the spontaneous $S O(10)$ gauge symmetry breaking and provides the necessary mixing among the $10_{H}$ and $\overline{126}_{H}$ weak doublet components needed to achieve (after electroweak symmetry breaking) a realistic fermion spectrum. The tiny VEV of the left-handed triplet component of

\footnotetext{
3 The fact that the fit always gives a hierarchical neutrino mass spectrum justifies a posteriori the neglect of the running of the neutrino parameters [37, 38].
}

$\overline{126}_{H}$ responsible for the type-II seesaw is induced via $210_{H}$ couplings as well. The model is minimal in the number of parameters, 26 altogether (soft SUSY breaking aside). The same number is found in the minimal supersymmetric standard model (MSSM) with right-handed neutrinos and it is much less than the number of parameters in the corresponding SUSY $S U(5)$ GUT.

Since none of the $126_{H}$ nor $210_{H}$ Higgs multiplets can couple to the $S O(10)$ matter bilinear $16_{m} \otimes 16_{m}$ at the renormalizable level, the Yukawa superpotential reads

$$
W_{Y}=16_{m}\left(Y_{10} 10_{H}+Y_{126} \overline{126}_{H}\right) 16_{m}
$$

while the $S O(10)$ Higgs sector is described by

$$
\begin{aligned}
W_{H}= & \frac{M_{210}}{4 !} 210_{H}^{2}+\frac{\lambda}{4 !} 210_{H}^{3}+\frac{M_{126}}{5 !} 126_{H} \overline{126}_{H} \\
& +\frac{\eta}{5 !} 126_{H} 210_{H} \overline{126}_{H}+M_{10} 10_{H}^{2} \\
& +\frac{1}{4 !} 210_{H} 10_{H}\left(\alpha 126_{H}+\bar{\alpha} \overline{126}_{H}\right) .
\end{aligned}
$$

The minimization of the scalar potential has been analyzed in great detail in Refs. [15, 29, 31]. The need of a careful study of the GUT scale potential in order to perform consistent predictions of the fermion mass textures is emphasized in these papers. The light MSSM-like Higgs doublets entering the low energy Yukawa potential are in general a superposition of corresponding doublet components of all the Higgs multiplets. The set of VEVs which describes the vacuum of the model is governed by one complex parameter $x$ [15]:

$$
\begin{aligned}
& \langle 1,1,1\rangle_{210}=-\frac{M_{210}}{\lambda} \frac{x\left(1-5 x^{2}\right)}{(1-x)^{2}}, \\
& \langle 15,1,1\rangle_{210}=-\frac{M_{210}}{\lambda} \frac{\left(1-2 x-x^{2}\right)}{(1-x)}, \\
& \langle 15,1,3\rangle_{210}=\frac{M_{210}}{\lambda} x, \\
& \langle\overline{10}, 1,3\rangle_{126}\langle 10,1,3\rangle_{\overline{126}}=\frac{2 M_{210}^{2}}{\eta \lambda} \frac{x(1-3 x)\left(1+x^{2}\right)}{(1-x)^{2}} .
\end{aligned}
$$

Avoiding D-term SUSY breaking requires $\langle\overline{10}, 1,3\rangle_{126}=\langle 10,1,3\rangle_{\overline{126}} \equiv v_{R}$. In Fig. 4 we depict the relevant $S O(10)$ breaking patterns together with the related $\mathrm{VEVs}$.

The various mass parameters in the GUT superpotential can be written as functions of $x$ as well. For instance

$$
M_{126}=M_{210} \frac{\eta}{\lambda} \frac{3-14 x+15 x^{2}-8 x^{3}}{(x-1)^{2}} .
$$

The mass parameter $M_{10}$ is determined by the minimal fine-tuning condition [13, 15] which drives the 


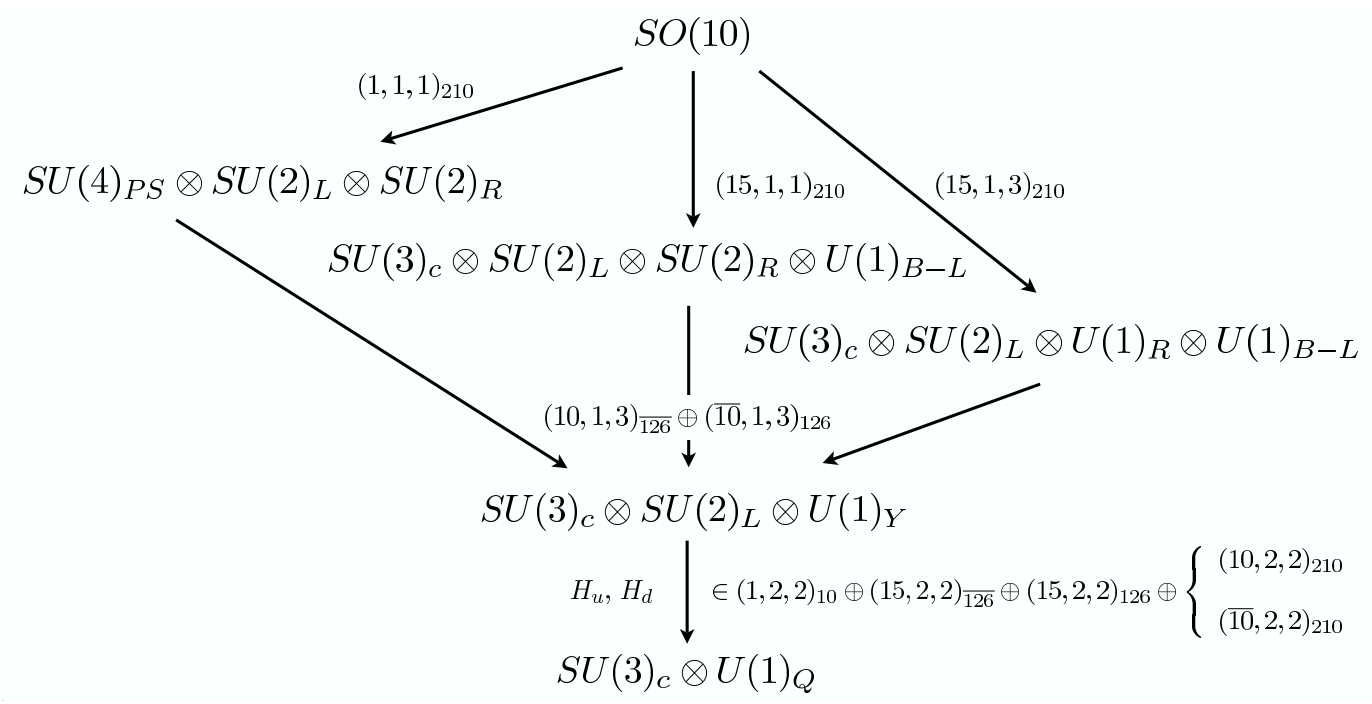

FIG. 4: Symmetry breaking patterns and related VEVs in the minimal SUSY SO(10). We do not display breaking chains involving intermediate $S U(5)$ symmetries, at odds with the proton decay constraints. Pati-Salam notation is used.

mass of two Higgs doublets down to the weak scale. The relevant formula reads

$$
M_{10}=M_{210} \frac{\alpha \bar{\alpha}}{2 \eta \lambda} \frac{p_{10}}{(x-1) p_{3} p_{5}},
$$

where $p_{n}$ are polynomials of $x$, see Refs. [15, 29]. The $S U(2)_{L}$ triplet mass, relevant for the type-II seesaw, can be written as

$$
M_{T}=M_{210} \frac{\eta}{\lambda} \frac{x\left(4 x^{2}-3 x+1\right)}{(x-1)^{2}}
$$

while the tiny induced $S U(2)_{L}$ triplet VEV is given by [13, 31]

$$
v_{L} \equiv\langle\overline{10}, 3,1\rangle_{\overline{126}}=\frac{\left(\alpha v_{u}^{10}+\sqrt{6} \eta v_{u}^{\overline{126}}\right) v_{u}^{210}}{M_{T}} .
$$

The electroweak VEV components $v_{u}$ 's are determined in terms of the electroweak scale and the $x$ parameter, c.f. Refs. [15, 29].

In the first part of the paper we learned that the best fit of the present data on fermion masses and mixing is obtained by a mixed contribution of type-I and type-II seesaws. The model independent analysis based only on the form of the Yukawa potential in Eq. (18) assumed the vacuum state to provide the required set of parameters, in particular the correct neutrino mass scale. In the following we examine thoroughly the question whether such a vacuum exists in the minimal renormalizable SUSY $S O(10)$ framework. The crucial issues are gauge coupling unification and the present limits on proton decay. Both instances in a low energy SUSY setting privilege a great desert scenario up to $10^{16} \mathrm{GeV}$. This requirement in turn tends to largely suppress the neutrino mass scale as obtained from type-I and/or type-II seesaw.

\section{A. Matter fermion fit in the minimal renormalizable SUSY $S O(10)$ model}

For our numerical analysis we adopt the parameterization and the phase convention of Ref. [29]. After minimal fine-tuning the Higgs potential can be described in terms of 8 real parameters:

$$
\begin{aligned}
& M_{210}, \operatorname{Re}(x), \operatorname{Im}(x), \alpha, \bar{\alpha},|\lambda|,|\eta|, \\
& \phi=\arg (\lambda)=-\arg (\eta) .
\end{aligned}
$$

For our purpose (fermion masses and symmetry breaking) without loss of generality we can set $\phi=0$. As emphasized in Refs. [15, 29], the parameter $x$ provides a systematic and effective way of describing the $S O(10)$ symmetry breaking patterns and the related fermion mass scales.

In the minimal SUSY $S O(10)$ framework under consideration the VEVs appearing in the fermion sum rules Eq. (11) are no longer free parameters (as assumed in the numerical analysis of Sec. III), but are functions of the Higgs potential parameters, c.f. Eq. (25). Using the results and notation of Ref. 29] one obtains the following expressions for the VEV combinations appearing in the fermion fit as defined in Eqs. (5) and (7):

$$
\begin{aligned}
f_{u} & =\frac{1}{4} \tan \beta \frac{N_{u}}{N_{d}}, \quad r=\frac{2 x p_{6}}{p_{2}} p_{5} \\
f_{\nu} & =\frac{v}{M_{210}} \tan \beta \sin \beta \alpha \sqrt{\frac{|\lambda|}{|\eta|}} \frac{N_{u}^{2}}{N_{d}}\left|f_{I I}(x)\right| \\
\xi & =\frac{1}{16} \frac{f_{I}(x)}{f_{I I}(x)} .
\end{aligned}
$$

Here $N_{u}, N_{d}$ are functions of the parameters in Eq. (25) given explicitly in the appendix of Ref. 29], 


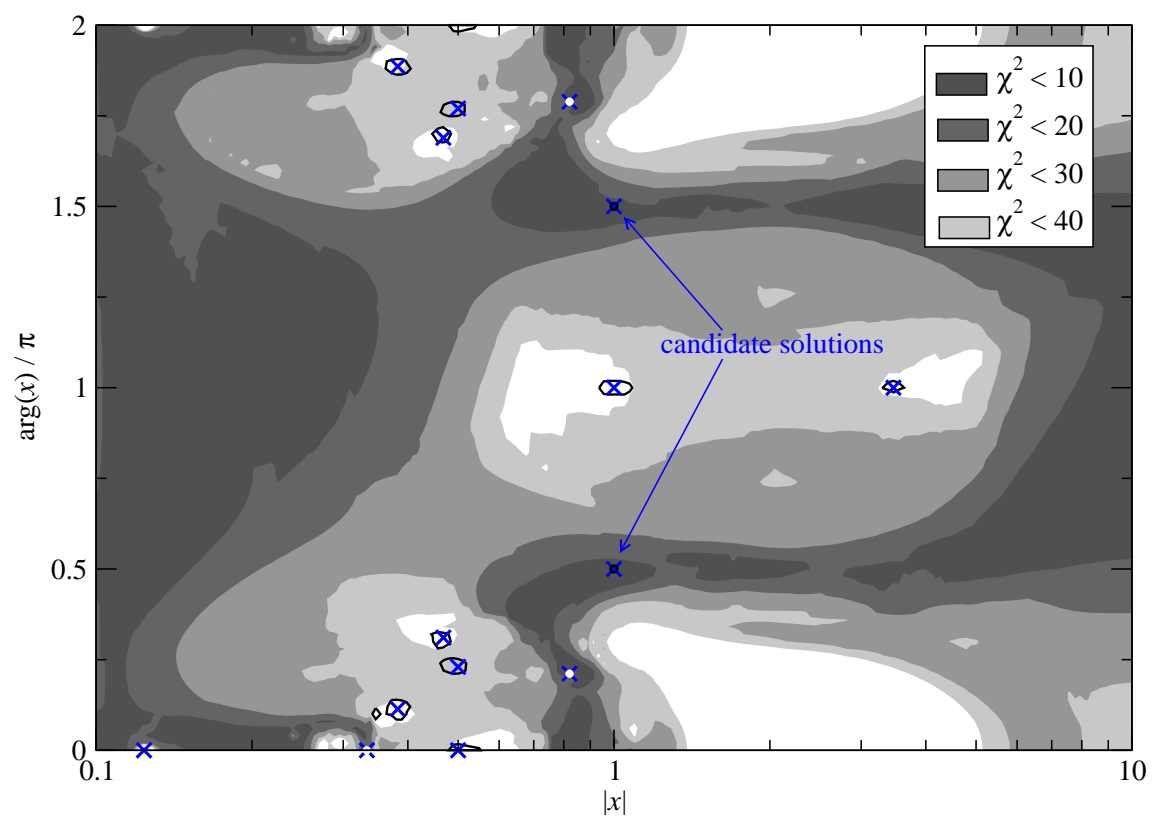

FIG. 5: $\chi^{2}$ contours of the fermion mass fit in the $x$ plane for the sample data in Table I. All scalar quartic couplings are set to one. The neutrino mass scale factor $f_{\nu}$ is treated as a free parameter. The black contour curves enclose the regions where $f_{\nu}(x) / f_{\nu}$ (fit) $>0.005$, where $f_{\nu}(x)$ is calculated from Eq. 26) and $f_{\nu}$ (fit) is the value obtained from the fermion fit. The cross symbols corresponds to points where either $f_{I}$ or $f_{I I}$ has a singular behaviour.

$v=174 \mathrm{GeV}$ is the electroweak scale, while

$$
f_{I}=\frac{2 p_{2} p_{5}}{p_{3} \sigma}, \quad f_{I I}=\frac{(x-1)(4 x-1) p_{3} q_{3}^{2} \sigma}{2 x p_{2} p_{5} q_{2}}
$$

where

$$
\sigma=\sqrt{\frac{2 x(1-3 x)\left(1+x^{2}\right)}{(1-x)^{2}}},
$$

and $p_{n}, q_{n}$ are polynomials of $x$ of order $n$ (see Tab. I in Ref. [29] ). ${ }^{4}$

We now address the question of whether a realistic fermion fit is possible, given the constraints from the Higgs sector encoded in Eqs. (26) which together with Eqs. (3), (4), and (6) lead to a highly constrained system of relations. First we note that not much freedom is left in adjusting the parameters $\alpha, \bar{\alpha}, \eta, \lambda$. This follows from the functional form of the VEVs in Eqs. (20), of the potential mass parameters, and of $f_{\nu}, N_{u}, N_{d}$, as well as from the requirement of perturbativity of the Higgs potential. Hence, in what follows we set $\alpha \sim \bar{\alpha} \sim \eta \sim$ $\lambda \sim O(1)$ [29]. Similar considerations suggest also $|x| \sim O(1)$, and therefore we first restrict our search to the range $0.1 \leq|x| \leq 10$ (deferring a discussion on the small and large $\bar{x}$ regimes to the end of the section).

\footnotetext{
${ }^{4}$ Notice that our parameter $\xi$ controls the relative weight of type-I and II seesaw, and is different from the $\xi(x)$ used in Ref. [29].
}

In order to identify candidate solutions we perform a scan in the complex $x$ plane by first fixing $\alpha=\bar{\alpha}=\eta=\lambda=1$. The $x$ dependence of $r$ and $\xi$ is taken into account according to Eq. (26), leaving however the neutrino mass normalization $f_{\nu}$ as a free parameter to be determined from the fit. Also $f_{u}$ becomes a free parameter, if $\tan \beta$ is allowed to vary. ${ }^{5}$

In Fig. 5 we show the $\chi^{2}$ contours of the fermion mass fit in the complex $x$ plane, where darker areas represent better fits of the fermion masses and mixings according to the $\chi^{2}$ values given. As it is visible from the figure we find large regions in the $x$ plane where a very good fit with $\chi^{2}<10$ can be obtained. The crucial question is whether within these regions it is possible to obtain also a consistent value of $f_{\nu}$. From Eq. (6) one finds that $f_{\nu}$ or $f_{\nu}|\xi|$ has to be of order $\sqrt{\Delta m_{31}^{2}} / m_{b} \sim 5 \times 10^{-11}$ to provide a neutrino mass scale required by oscillation data, in agreement with the values reported in Tab. II] In contrast, from Eq. (26) one finds a natural size of

$$
f_{\nu} \sim \frac{v \tan \beta}{M_{210}} \sim 5 \times 10^{-13}\left(\frac{\tan \beta}{55}\right)
$$

for $M_{210}=2 \times 10^{16} \mathrm{GeV}$. This estimate illustrates

\footnotetext{
5 In the following we will consider $\tan \beta$ as a free parameter within the range $10 \lesssim \tan \beta \lesssim 55$. We may still keep the data in Table [1 as reference GUT values for the fermion parameters, since changing $\tan \beta$ in the indicated range has a small impact on the running [35].
} 


\begin{tabular}{|c|c|c|}
\hline parameter & best fit & $\lambda, \alpha, \bar{\alpha}$ fixed \\
\hline$|x|$ & 1.0000002207 & 1.00000009837 \\
\hline $\arg (x) / \pi$ & 0.4999999781 & 0.4999999815 \\
\hline$\eta$ & 0.3191 & 0.3191 \\
\hline$\lambda$ & 1.5642 & 1 \\
\hline$\alpha$ & 2.9455 & 1 \\
\hline $\bar{\alpha}$ & 4.0332 & 1 \\
\hline$M_{210}[\mathrm{GeV}]$ & $2.000 \times 10^{16}$ & $2.000 \times 10^{16}$ \\
\hline $\tan \beta$ & 41.15 & 45.88 \\
\hline$|\xi|$ & $7.476 \times 10^{4}$ & $1.513 \times 10^{5}$ \\
\hline $\arg (\xi) / \pi$ & -0.60492 & -0.53116 \\
\hline$|r|$ & 1.955 & 1.955 \\
\hline $\arg (r) / \pi$ & -0.2568 & -0.2568 \\
\hline$f_{u}$ & 12.28 & 11.47 \\
\hline$f_{\nu}$ & $7.077 \times 10^{-17}$ & $3.745 \times 10^{-17}$ \\
\hline$\langle 1,1,1\rangle[\mathrm{GeV}]$ & $3.836 \times 10^{16}$ & $6.000 \times 10^{16}$ \\
\hline$\langle 15,1,1\rangle[\mathrm{GeV}]$ & $2.557 \times 10^{16}$ & $4.000 \times 10^{16}$ \\
\hline$\langle 15,1,3\rangle[\mathrm{GeV}]$ & $1.279 \times 10^{16}$ & $2.000 \times 10^{16}$ \\
\hline$\langle 10,1,3\rangle[\mathrm{GeV}]$ & $3.423 \times 10^{13}$ & $3.009 \times 10^{13}$ \\
\hline$M_{P G}[\mathrm{GeV}]$ & $4.135 \times 10^{10}$ & $2.043 \times 10^{10}$ \\
\hline$M_{T}[\mathrm{GeV}]$ & $8.655 \times 10^{15}$ & $1.354 \times 10^{16}$ \\
\hline observable & pred. & pred. \\
\hline$m_{d}[\mathrm{MeV}]$ & $0.3435 \quad-\mathbf{2 . 2}$ & 0.3373 \\
\hline$m_{s}[\mathrm{MeV}]$ & 27.91 & 26.36 \\
\hline$m_{b}[\mathrm{MeV}]$ & $1065 \quad 0.038$ & 1112 \\
\hline$m_{u}[\mathrm{MeV}]$ & $0.5596 \quad 0.038$ & 0.5639 \\
\hline$m_{c}[\mathrm{MeV}]$ & 212.8 & 213.1 \\
\hline$m_{t}[\mathrm{MeV}]$ & $82090-0.21$ & $79550 \quad-0.19$ \\
\hline $\sin \phi_{23}^{\mathrm{CKM}}$ & $0.0351-0.039$ & $0.0350-0.065$ \\
\hline $\sin \phi_{13}^{\mathrm{CKM}}$ & 0.00325 & 0.00329 \\
\hline $\sin \phi_{12}^{\mathrm{CKM}}$ & $0.2244 \quad 0.033$ & 0.2244 \\
\hline$\delta_{\mathrm{CKM}}\left[{ }^{\circ}\right]$ & $64.32 \quad 0.31$ & 70.10 \\
\hline $\sin ^{2} \theta_{23}^{\mathrm{PMNS}}$ & $0.4893-0.16$ & $0.5002 \quad 0.0035$ \\
\hline $\sin ^{2} \theta_{13}^{\mathrm{PMNS}}$ & 0.0133 & 0.01085 \\
\hline $\sin ^{2} \theta_{12}^{\text {PMNS }}$ & $0.3007 \quad-0.37$ & $0.2953 \quad-0.59$ \\
\hline$\Delta m_{21}^{2}\left[10^{-5} \mathrm{eV}^{2}\right]$ & $7.875-0.082$ & -0.13 \\
\hline$\Delta m_{31}^{2}\left[10^{-3} \mathrm{eV}^{2}\right]$ & $2.199-0.002$ & 2.274 \\
\hline$m_{1} / \sqrt{\Delta m_{21}^{2}}$ & 0.3351 & 0.3289 \\
\hline$\chi^{2}$ & 7.25 & 7.30 \\
\hline
\end{tabular}

TABLE III: Parameter values and outcomes for the best fit solution, $x \simeq i$. The reference mass parameter $M_{210}$ is set at a typical MSSM GUT scale. The charged lepton masses are given in Eq. (10). Deviations above $2 \sigma$ in the fermion mass data are highlighted in boldface. The final $\chi^{2}$ is the sum of the squares of the numbers in the "pull" column.

that in the framework under consideration a gap of about 2 orders of magnitude exists between the generic prediction of the neutrino masses and the scale required by the data. We have verified this expectation numerically and for most part of the complex $x$ plane shown in Fig. 5 the value of $f_{\nu}(x)$ according to Eq. (26) is 2 to 4 orders of magnitude smaller than the value of $f_{\nu}$ required by the fit (denoted by $f_{\nu}($ fit $\left.)\right)$.
The only way left to obtain the needed neutrino mass scale is to consider special points in the $x$ plane, where either $\left|f_{I I}(x)\right|$ or $\left|f_{I}(x)\right|$ becomes large because of roots in the denominators of Eq. (27). These singularities are marked in Fig. 5 by the crosses. The closed black contours correspond to the regions where $f_{\nu}(x) / f_{\nu}$ (fit) $>0.005$. We find that only close to the singularities $f_{\nu}$ can be large enough to provide the required neutrino mass scale. On the other hand, it appears that most of the singularities drop into regions where the fermion mass fit is poor. We carefully checked numerically the regions close to the relevant singularities and came to the conclusion that only in the near neighborhood of $x= \pm i$ a realistic fit of the matter sector is possible. Helpful discussions of specific points and limiting cases in the $x$ complex plane based on analytical considerations can be found in Refs. [15, 29].

In Tab. III we report the best fit solution we found. The absolute value and phase of $x$ are tuned at the level of $10^{-7}$ in the close neighborhood of $i$. The "symmetric" solution $x=-i$ exhibits to quite similar features. In the "best fit" column we have optimized the parameters $\alpha, \bar{\alpha}, \eta, \lambda$, whereas in the third column we have taken $\alpha=\bar{\alpha}=\lambda=1$. As expected, changing these parameters has very little impact on the fit, as it is evident from the comparison of the second and third column. Requiring in addition $\eta=1$ gives a very similar result. The neutrino mass matrix is dominated by type-I seesaw since $\sigma=0$ for $x= \pm i$, which implies $f_{I} \rightarrow \infty$ and $f_{I I}=0$, and hence $\xi \rightarrow \infty$, see Eqs. (27) and (26). From the data in the table one finds $f_{\nu}|\xi| \sim 10^{-11}$, which is the correct value to provide the required neutrino mass scale. The table shows that a very good fit of all quark and lepton mass and mixing parameters (including the neutrino sector) is obtained, with just a $2.2 \sigma$ pull for $m_{d}$ that is acceptable in view of the systematic uncertainities entering the light quark mass determinations.

\section{B. Gauge couplings unification and proton decay for $x \simeq \pm i$}

What remains to be checked is whether the best fit solution satisfies unification and proton decay constraints. For $x= \pm i$ the $S O(10)$ gauge symmetry is broken ${ }^{6}$ to $G_{3211} \equiv S U(3)_{C} \times S U(3)_{L} \times U(1)_{R} \times$ $U(1)_{B-L}$ by the VEV of $(15,1,3)_{210}$ (compare Fig. 14 and Refs. [15, 29]). The calculation of the particle

6 We neglect the tiny hierarchy among the VEVs of $(1,1,1)_{210},(15,1,1)_{210}$, and $(15,1,3)_{210}$ as all of them are confined for $x= \pm i$ within an interval of less than one order of magnitude, c.f. Eq. (20) and Tab. III 


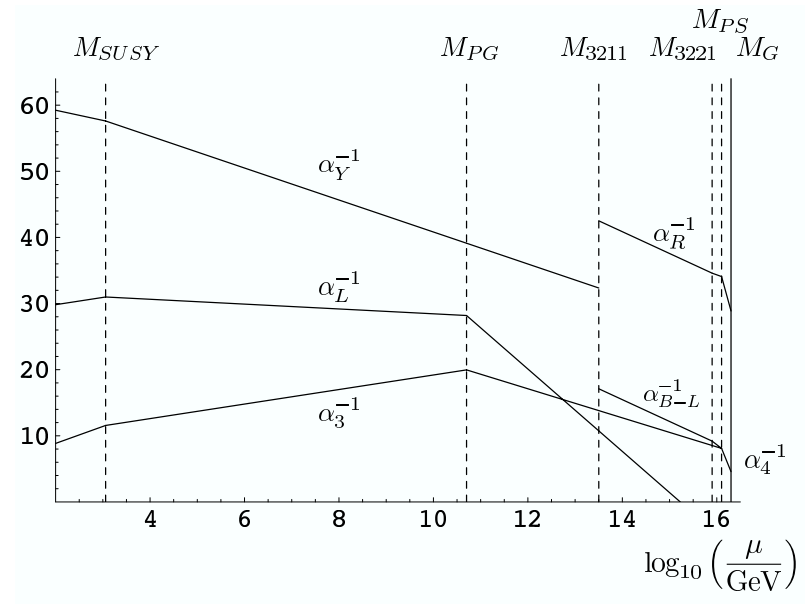

FIG. 6: Running gauge coupling constants in the setup of Table III We have $M_{\text {SUSY }}=1 \mathrm{TeV}, M_{P G}=M_{(8,3,0)}$, $M_{3211} \equiv\langle 10,1,3\rangle, M_{3221} \equiv\langle 15,1,1\rangle, M_{P S} \equiv\langle 1,1,1\rangle$, and $M_{\mathrm{GUT}} \equiv M_{210}=2 \times 10^{16} \mathrm{GeV}$.

spectrum shows that in this limit a set of unmixed states that transform as $(8,3,0,0)$ under $G_{3211}$ (or $(8,3,0)$ under the SM gauge group) remains light. In the exact limit $x= \pm i$ these Goldstone states are massless because of the spontaneous breaking of accidental global symmetries of the Higgs potential. Upon breaking the $G_{3211}$ gauge symmetry to the SM via the $\operatorname{VEVs}^{7}\langle 10,1,3\rangle_{\overline{126}}=\langle\overline{10}, 1,3\rangle_{126}=v_{R}(\equiv$ $\left.M_{3211}\right)$ they acquire a mass $M_{P G}$ of the order of $M_{3211}^{2} / M_{P S}$, where $M_{P S} \equiv\langle 1,1,1\rangle_{210}$ is the PatiSalam scale. According to Ref. [15] the expression for the Pseudo-Goldstone mass is

$$
M_{P G}=-4 M_{210} \frac{(2 x-1)\left(x^{2}+1\right)}{(x-1)^{2}},
$$

which is exactly zero for $x= \pm i$. In our best fit case reported in Tab. [III) we find $M_{3211} \sim 10^{13} \mathrm{GeV}$, while $M_{P S} \sim 10^{16} \mathrm{GeV}$. One therefore expects $M_{P G} \sim$ $10^{10} \mathrm{GeV}$, as we consistently find (see table).

In spite of the fact that these states are much lighter than the GUT scale they do not affect proton decay because of their zero $B-L$ charge. However, they do affect heavily the running of the gauge couplings (they transform as adjoint representations of $S U(3)_{c}$ and $\left.S U(2)_{L}\right)$. Fig. [6] shows how dramatically the running is affected. The unification of the gauge couplings is completely spoiled, with the coupling constant of $S U(2)_{L}$ diverging below the GUT scale. Hence, the only solution found providing a realistic fit to all fermion masses and mixing parameters has to be discarded because of the dramatic failure of the

\footnotetext{
7 More precisely, the quantum numbers of the states responsible for this symmetry breaking step are: $(1,1, \pm 1, \mp 2)^{3211} \in(1,1,3, \mp 2)^{3221} \in(10,1,3) \overline{126} \oplus$ h.c.
}

gauge couplings unification. The minimal renormalizable SUSY $S O(10)$ scenario seems therefore failing to provide a realistic description of the low energy world. In general, if right-handed neutrinos trasform non-trivially under the GUT gauge symmetry, the minimum neutrino mass scale required by oscillation data implies via type I (or type II) seesaw the presence of states at intermediate mass scales [29, 30], that spoil the successful unification within the MSSM.

In passing let us stress that the example discussed above shows quite clearly that the naive argument of setting all relevant particle states at the scale of the symmetry breaking step may be far from correct. One should always be aware that much lighter states may appear in the spectrum as a consequence of the spontaneous breaking of accidental (would-be) symmetries of the scalar potential [4].

\section{Large/small $|x|$ regime}

Before conclusively rejecting the minimal renormalizable SUSY $S O(10)$ scenario we must make sure that the domain $0.1 \lesssim|x| \lesssim 10$ considered in the previous analysis covers the physically relevant region. Indeed, the shape of the $\chi^{2}$ contours in Fig. 5 suggests that all the matter fermion data might be well reproduced in areas outside the considered region. Let us give simple semianalytic arguments against this option.

By observing that in the large $x$ regime all $210_{H}$ VEVs in Eq. (20) scale as $M_{210}|x| / \lambda$ and proton decay forces us to maintain most of the GUT states above $M_{\mathrm{GUT}} \simeq 10^{16} \mathrm{GeV}$, we must require

$$
\frac{M_{210}|x|}{\lambda} \simeq 10^{16} \mathrm{GeV} .
$$

In the large $x$ limit (and for large $\tan \beta$ ) from Eqs. (26), (27) and the expressions for $N_{u}, N_{d}$ given in Ref. 29] one finds for the neutrino mass scales

$$
f_{\nu}|\xi| \propto \frac{v}{M_{210}|x|} \tan \beta, f_{\nu} \propto \frac{v}{M_{210}|x|} \frac{\tan \beta}{|x|}
$$

in the type-I and type-II seesaw cases respectively. By requiring $M_{210}|x| \simeq$ const (see Eq. (31D) these relations show that also for large $x$ the neutrino mass scale cannot be efficiently enhanced over its natural value $v^{2} / M_{\mathrm{GUT}}$, and accordingly our numerical calculation yields values of $f_{\nu}$ of about four orders of magnitude too small in the region $|x|>10$. In passing let us remark that in the $|x| \gg 1$ regime there appear a number of states with masses around $M_{210}<M_{G U T}$ that again spoil gauge unification, albeit not affecting proton decay (see Ref. [15]). 
In the small $|x|$ regime one finds for both seesaw types

$$
f_{\nu}, f_{\nu}|\xi| \propto \frac{v}{M_{210}|x|} \tan \beta \sqrt{|x|} .
$$

Once again taking into account Eqs. (20131) and the fact that scalar couplings are bound to vary in a narrow range by perturbativity on one side and mass scale splittings on the other, no substantial enhancement of the neutrino mass can be expected. Our $\chi^{2}$ fit does confirm numerically these expectations.

We therefore conclusively show, independently confirming the conclusions of Ref. [30], that the minimal renormalizable SUSY $S O(10)$ setup in spite of noteworthy and impressive features in reproducing the observed flavor textures fails in reproducing the neutrino mass scale. Generally, in a renormalizable framework, type I and type II seesaws call for intermediate scales that spoil (SUSY) gauge unification.

\section{CONCLUSIONS}

In the present paper we studied in great detail the fit of quark and lepton masses and mixing data within a class of supersymmetric $S O(10)$ grand unified models with a minimal renormalizable Yukawa sector based on one $10_{H}$ and one $\overline{126}_{H}$ Higgs representation. A systematic optimization of the relevant $\chi^{2}$ function has been performed, complementary to random parameter searches applied in previous studies. We have shown that for a comparable size of the type-I and type-II seesaw terms, an excellent fit to the fermion data can be obtained, where all observables (including the CKM CP phase) are fitted within less than 0.4 standard deviations. Solutions based on pure type-I and type-II seesaw have been discussed as well, and we have identified a new class of possible solutions corresponding to a singular behaviour of the type-II Majorana mass matrix. The corresponding predictions for the neutrino parameters have been investigated in detail.

In the second part of the paper these general results were confronted with the additional restrictions emerging in the minimal renormalizable SUSY $S O(10)$ scenario from the model vacuum as well as from proton decay and unification constraints. We identified a very limited (and fine-tuned) area in the parameter space where, given the constraints from the minimization of the Higgs potential, a fit to the quark and lepton masses and mixing parameters is possible. However, all solutions providing a realistic fit to the matter fermion data had to be discarded because of the failure in reproducing the strenght of the low energy gauge couplings. In
GUTs that embed non trivially right-handed neutrinos the absolute neutrino mass scale required by oscillation data generally implies via type I and/or type II seesaws the presence of states at intermediate mass scales, that are likely to spoil the successful unification achieved in the MSSM. Our analysis did in fact exclude, in the case of the minimal SUSY $S O(10)$ model, the existence of (fine tuned) exceptional solutions.

The minimal renormalizable SUSY $S O(10)$ scenario based on one $10_{H}$, one $\overline{126}_{H}$, one $126_{H}$, and one $210_{H}$ Higgs representations is conclusively rejected. Our discussion suggests that either one attempts to enlarge the scalar (Yukawa) potential in order to soften some of the parameter correlations on the vacuum, or non-renormalizable terms are included providing effectively the needed depletion of the seesaw scale. Alternatively, one is let to consider the intriguing option of a non-supersymmetric GUT scenario, where intermediate scales, preferred by neutrino data, are needed anyway by gauge coupling unification. Beauty and loss do strike our deepest chords. As the poet says:

Is that time dead? lo! with a little rod

I did but touch the honey of romance-

And must I lose a soul's inheritance?

(Oscar Wilde, Helas, 1881).

\section{Acknowledgments}

We thank W. Grimus for useful discussions, C.S. Aulakh and R.N. Mohapatra for comments on the manuscript, and T. Shindou for providing $\mathrm{C}++$ routines used in this analysis. The work of T.S. is supported by a Marie Curie Intra-European Fellowship within the $6^{\text {th }}$ European Community Framework Program. The work of S.B. is partially supported by MIUR and the RTN European Program MRTN-CT-2004-503369. One of us (M.M.) is grateful to SISSA and CERN for the hospitality during the preparation of the manuscript.

\section{Note added}

The arXiv version 4 of this paper corrects three entries in Table III, namely the value of $\eta$ in the third column, equal to that in the second column, and the two values of $f_{\nu}$ that were off by a factor two. We thank Borut Bajc, Ilja Dorsner and Miha Nemevsek for carefully checking our results and spotting the typos. 
[1] H. Georgi, in Particles and Fields, edited by C. E. Carlson (AIP, New York, 1975); H. Fritzsch and P. Minkowski, Unified Interactions Of Leptons And Hadrons, Annals Phys. 93 (1975) 193.

[2] P. Minkowski, $M u \rightarrow E$ Gamma At A Rate Of One Out Of 1-Billion Muon Decays?, Phys. Lett. B 67, 421 (1977); M. Gell-Mann, P. Ramond and R. Slansky, Complex Spinors And Unified Theories, In Supergravity, P. van Nieuwenhuizen and D.Z. Freedman (eds.), North Holland Publ. Co., 1979, p. 315; T. Yanagida, Horizontal Gauge Symmetry And Masses Of Neutrinos, In Proc. Workshop on the Baryon Number of the Universe and Unified Theories, O. Sawada and A. Sugamoto (eds.), Tsukuba, Japan, 13-14 Feb. 1979, p. 95; S.L. Glashow, The Future Of Elementary Particle Physics, HUTP-79A059 In Proc. Cargese 1979 Quarks and Leptons, p. 687 ; R.N. Mohapatra and G. Senjanović, Neutrino Mass And Spontaneous Parity Nonconservation, Phys. Rev. Lett. 44, 912 (1980).

[3] M. Magg and C. Wetterich, Neutrino Mass Problem And Gauge Hierarchy, Phys. Lett. B 94, 61 (1980); G. Lazarides, Q. Shafi and C. Wetterich, Proton Lifetime And Fermion Masses In An SO(10) Model, Nucl. Phys. B 181, 287 (1981); R. N. Mohapatra and G. Senjanović, Neutrino Masses And Mixings In Gauge Models With Spontaneous Parity Violation, Phys. Rev. D 23, 165 (1981).

[4] C. S. Aulakh and R. N. Mohapatra, Implications Of Supersymmetric SO(10) Grand Unification, Phys. Rev. D 28, 217 (1983).

[5] J. C. Pati and A. Salam, Lepton Number As The Fourth Color, Phys. Rev. D 10 (1974) 275.

[6] K. S. Babu and R. N. Mohapatra, Predictive neutrino spectrum in minimal $S O(10)$ grand unification, Phys. Rev. Lett. 70 (1993) 2845 hep-ph/9209215.

[7] L. Lavoura, Predicting the neutrino spectrum in minimal $S O(10)$ grand unification, Phys. Rev. D 48 (1993) 5440 hep-ph/9306297.

[8] C. S. Aulakh, B. Bajc, A. Melfo, A. Rasin and G. Senjanovic, $S O(10)$ theory of R-parity and neutrino mass, Nucl. Phys. B 597 (2001) 89 hep-ph/0004031.

[9] T. Fukuyama and N. Okada, Neutrino oscillation data versus minimal supersymmetric $S O(10)$ model, JHEP 0211 (2002) 011 hep-ph/0205066.

[10] B. Bajc, G. Senjanovic and F. Vissani, b - tau unification and large atmospheric mixing: A case for non-canonical see-saw, Phys. Rev. Lett. 90 (2003) 051802 hep-ph/0210207.

[11] H. S. Goh, R. N. Mohapatra and S. P. Ng, Minimal SUSY SO(10), b tau unification and large neutrino mixings, Phys. Lett. B 570, 215 (2003) hep-ph/0303055.

[12] T. Fukuyama, T. Kikuchi and N. Okada, Lepton flavor violating processes and muon $\mathrm{g}$ - 2 in minimal supersymmetric SO(10) model, Phys. Rev. D 68 (2003) 033012 hep-ph/0304190;

[13] C. S. Aulakh, B. Bajc, A. Melfo, G. Senjanovic and F. Vissani, The minimal supersymmetric grand unified theory, Phys. Lett. B 588 (2004) 196 hep-ph/0306242.
[14] H. S. Goh, R. N. Mohapatra and S. P. Ng, Minimal SUSY SO(10) model and predictions for neutrino mixings and leptonic CP violation, Phys. Rev. D 68, 115008 (2003) hep-ph/0308197.

[15] B. Bajc, A. Melfo, G. Senjanovic and F. Vissani, The minimal supersymmetric grand unified theory. I: Symmetry breaking and the particle spectrum, Phys. Rev. D 70, 035007 (2004) hep-ph/0402122.

[16] B. Bajc, G. Senjanovic and F. Vissani, Probing the nature of the seesaw in renormalizable $S O(10)$, Phys. Rev. D 70 (2004) 093002 hep-ph/0402140.

[17] B. Dutta, Y. Mimura and R. N. Mohapatra, $C K M$ $C P$ violation in a minimal $S O(10)$ model for neutrinos and its implications, Phys. Rev. D 69 (2004) 115014 hep-ph/0402113.

[18] H. S. Goh, R. N. Mohapatra and S. Nasri, SO(10) symmetry breaking and type II seesaw, Phys. Rev. D 70, 075022 (2004) arXiv:hep-ph/0408139.

[19] C. S. Aulakh and A. Girdhar, $S O(10)$ a la PatiSalam, Int. J. Mod. Phys. A 20, 865 (2005) hep-ph/0204097; SO(10) MSGUT: spectra, couplings and thresholds effects, Nucl. Phys. B 711, 275 (2005) hep-ph/0405074.

[20] T. Fukuyama, A. Ilakovac, T. Kikuchi, S. Meljanac and N. Okada, Higgs masses in the minimal SUSY SO(10) GUT, Phys. Rev. D 72 (2005) 051701 hep-ph/0412348.

[21] C. S. Aulakh, Consistency of the minimal supersymmetric GUT spectra, Phys. Rev. D 72, 051702 (2005).

[22] T. Fukuyama, T. Kikuchi and T. Osaka, Nonthermal leptogenesis and a prediction of inflaton mass in a supersymmetric SO(10) model, JCAP 0506 (2005) 005 hep-ph/0503201.

[23] S. Bertolini, M. Frigerio and M. Malinsky, Fermion masses in a SUSY SO(10) model with type II seesaw: A non-minimal predictive scenario, Phys. Rev. D 70, 095002 (2004) hep-ph/0406117.

[24] S. Bertolini and M. Malinsky, On CP violation in a minimal renormalizable SUSY SO(10) model and beyond, Phys. Rev. D 72, 055021 (2005) hep-ph/0504241.

[25] K. S. Babu and C. Macesanu, Neutrino masses and mixings in a minimal SO(10) model, Phys. Rev. D 72 (2005) 115003 hep-ph/0505200.

[26] B. Dutta, Y. Mimura and R. N. Mohapatra, Neutrino mixing predictions of a minimal SO(10) model with suppressed proton decay, Phys. Rev. D 72, 075009 (2005) hep-ph/0507319.

[27] H. S. Goh, R. N. Mohapatra, S. Nasri and S. P. Ng, Proton decay in a minimal SUSY SO(10) model for neutrino mixings, Phys. Lett. B 587, 105 (2004) hep-ph/0311330.

[28] T. Fukuyama, A. Ilakovac, T. Kikuchi, S. Meljanac and N. Okada, Detailed analysis of proton decay rate in the minimal supersymmetric $S O(10)$ model, JHEP 0409, 052 (2004) hep-ph/0406068; General formulation for proton decay rate in minimal supersymmetric SO(10) GUT, Eur. Phys. J. C 42 (2005) 191 hep-ph/0401213.

[29] B. Bajc, A. Melfo, G. Senjanovic and F. Vissani, Fermion mass relations and the structure of the light 
Higgs in a supersymmetric $S O(10)$ theory, Phys. Lett. B, 634 (2006) 272 hep-ph/0511352.

[30] C. S. Aulakh and S. K. Garg, MSGUT: From Bloom to Doom, hep-ph/0512224.

[31] C. S. Aulakh, MSGUTs from germ to bloom: Towards falsifiability and beyond, hep-ph/0506291.

[32] C. S. Aulakh, Fermion mass hierarchy in the Nu MSGUT. I: The real core, hep-ph/0602132.

[33] L. Lavoura, H. Kühböck and W. Grimus, Chargedfermion masses in $S O(10):$ Analysis with scalars in 10+120, hep-ph/0603259.

[34] R. N. Mohapatra and A. Y. Smirnov, Neutrino mass and new physics, hep-ph/0603118.

[35] C. R. Das and M. K. Parida, New formulas and predictions for running fermion masses at higher scales in SM, 2HDM, and MSSM, Eur. Phys. J. C 20 (2001) 121 hep-ph/0010004.

[36] Particle Data Group, S. Eidelman et al., Phys. Lett. B 592 (2004) 1 and 2005 partial update for the 2006 edition available on http://pdg.lbl.gov/

[37] S. Antusch, J. Kersten, M. Lindner and M. Ratz, Running neutrino masses, mixings and CP phases: Analytical results and phenomenological consequences, Nucl. Phys. B 674, 401 (2003) hep-ph/0305273.
[38] S. Antusch, J. Kersten, M. Lindner, M. Ratz and M. A. Schmidt, Running neutrino mass parameters in see-saw scenarios, JHEP 0503, 024 (2005) hep-ph/0501272.

[39] M. Maltoni, T. Schwetz, M. A. Tortola and J. W. F. Valle, Status of global fits to neutrino oscillations, New J. Phys. 6 (2004) 122 hep-ph/0405172;

[40] T. Schwetz, Neutrino oscillations: Current status and prospects, Acta Phys. Polon. B 36 (2005) 3203 hep-ph/0510331.

[41] See for instance, W. H. Press et al., Numerical Recipes in C: The Art of Scientific Computing, Cambridge University Press 1992.

[42] S. Antusch, P. Huber, J. Kersten, T. Schwetz and $\mathrm{W}$. Winter, Is there maximal mixing in the lepton sector?, Phys. Rev. D 70 (2004) 097302 hep-ph/0404268.

[43] P. Huber, M. Lindner, M. Rolinec, T. Schwetz and W. Winter, Prospects of accelerator and reactor neutrino oscillation experiments for the coming ten years, Phys. Rev. D 70 (2004) 073014 hep-ph/0403068. 